\title{
Improvement of fluid balance monitoring through education and rationalisation
}

\author{
Masaki Vincent, Thabo Mahendiran \\ Musgrove Park Hospital, Taunton and Somerset Trust, United Kingdom
}

\begin{abstract}
Fluid input/output charts in hospital inpatients are a valuable source of information for doctors reviewing intravenous fluid prescription, but are notorious for being incomplete and inaccurate. Lack of awareness of the importance of fluid balance amongst nursing staff and an excess of unnecessary monitoring are two factors contributing to the problem. We conducted a quality improvement project on the respiratory ward in a large district general hospital aiming to specifically address these two factors. Pre-intervention audit showed that only 53\% of input/output monitoring was clinically indicated, with an average chart completion of $50 \%$. Using e-Learning and verbal presentation to raise awareness around fluid balance, we implemented a new system whereby daily medical review of charts lead to rationalisation of monitoring. Postintervention audit showed a 93\% reduction in unnecessary monitoring, with corresponding increases in completion (40\%) and accuracy (48\%) of remaining charts. In conclusion, education has enabled a culture change on the ward that has drastically increased the quality of fluid balance monitoring.
\end{abstract}

\section{Problem}

Fluid balance monitoring through the use of input/output charts in hospital inpatients has been notoriously inaccurate for decades[1-3] due to several factors.

Some factors will be difficult to change: input/output monitoring is an inherently difficult task, especially in confused or incontinent patients. It is time consuming and therefore difficult to perform accurately on busy hospital wards, without the high staffing ratios available in critical care settings.

Some factors, however, can be changed. There are no clear rules determining when to monitor fluid balance, with many charts being started in patients who don't require daily input/output monitoring, thus leaving nursing staff less time to focus on those that are important. Unnecessary charts are ignored by medical teams, leading nursing staff to perceive the task as futile. There is no guidance from the medical team regarding when to stop monitoring, with most charts being continued at midnight by nursing staff who haven't communicated with the day team. Finally, there is a lack of awareness and education around the importance of fluid status, especially in the healthcare assistant population, who often carry out the bulk of input/output monitoring.

\section{Background}

Junior doctors are often called to see patients to review intravenous fluid or diuretic prescription. Accurate knowledge of fluid status is crucial to making these decisions.[4] Physical examination and measurement of vital signs may be helpful, but abnormal signs only start to appear once fluid status is grossly abnormal, hence the utility of input/output charts. Inaccurate input/output monitoring may lead to late detection of fluid status abnormalities and poor decision- making.

Poor fluid prescribing leads to disturbances of volume status, which increase the risk of many common complications of inpatient stay.[5] Hypervolaemia may cause pulmonary oedema and increase the risk of hospital-acquired pneumonia, or may cause peripheral oedema, increasing the risk of pressure sores and impaired wound healing. Hypovolaemia, in turn, is a wellrecognised cause of acute kidney injury, with its associated complications.

Poor fluid prescribing may also cause disturbances of electrolyte status, such as hypernatraemia and hypokalaemia from excessive administration of intravenous normal saline.

Accurate documentation of fluid status enabling judicious fluid prescribing is therefore fundamental to safe inpatient care.

\section{Baseline measurement}

We performed a snapshot audit across four general medical wards $(\mathrm{n}=117$ patients). For each patient, we assessed:

a. Whether daily fluid balance monitoring was happening.

b. Whether daily fluid balance monitoring was clinically indicated. We used a list of indications agreed upon with two General Medicine consultants (eg sepsis, AKI, fluid overload states, acutely unwell, etc).

c. Whether "totals" boxes had been completed (eg 12 hours total for oral input, 24 hours total for oral input, 12 hours total for IV input, etc). We defined "chart completion rate" as the ratio of the number of "totals" boxes that had been filled in, divided by the number of "totals" boxes that should have been filled in. 
d. Whether "totals" boxes were accurate. We defined a sum as "accurate" if the error in the final sum was inferior to $10 \%$ of the sum itself.

This initial snapshot showed that:

a. $67 \%$ of patients were on input/output monitoring

b. Of all patients on input/output monitoring, it was only clinically relevant in $53 \%$

c. Average chart completion rate was $50 \%$

d. Average chart accuracy was $41 \%$

In addition to this audit, we designed an online survey for Nurses and Healthcare assistants $(n=43)$ to assess their views:

- $72 \%$ said that input/output charts were being started in too many patients unnecessarily

- $91 \%$ said that monitoring was being continued for longer than necessary during inpatient stay

- $84 \%$ said that having more guidance from the medical team regarding monitoring would be helpful

- $67 \%$ said that more education on fluid balance would be helpful

\section{Design}

We had two main interventions. Firstly, we designed a new fluid balance monitoring chart (Figure 1) with an "Indications" box where the ward doctor had to justify why fluid balance monitoring was clinically relevant in that patient. The doctor then had to sign the "Continue?" box, determining whether or not monitoring should be continued.

This system had two purposes. Firstly, it forced the reviewing doctor to actively think about the relevance of fluid balance on a daily basis, leading to a pro-active decision to continue or stop monitoring. This is in contrast to the previous situation, where doctors were passive and most charts were continued by nursing staff "by default". Secondly, the chart allowed nursing staff to see a clear justification for the task they were being asked to perform.

Secondly, we designed an e-learning module for nurses and healthcare assistants (figure 2), which explained why fluid balance monitoring was important, and in whom. The module contained a mix of didactic-style teaching slides and interactive slides with case studies and multiple choice questions.

\section{Strategy}

PDSA cycle 1: We implemented our new chart across four general medical wards at Musgrove Park Hospital. This initial launch was difficult: many nurses and HCAs resented being faced with an unfamiliar chart. Furthermore, due to shift patterns and the size of the staffing pool, it was difficult to find a time to speak to all members of staff in order to explain the project. We did not have enough manpower to coordinate the project across multiple wards at once, so decided to focus on one ward, the Respiratory Ward.

PDSA cycle 2: In order to raise awareness of our project on the Respiratory Ward, we displayed posters around nursing areas on the ward. Since it was difficult to organise an information session that all staff could attend, members of our team regularly attended nursing handover in order to publicise the project with a short presentation. As exposure and awareness grew, our charts came into full use.

PDSA cycle 3: With support from the ward Sisters, we launched our newly designed e-Learning module. Feedback from nursing staff showed that they needed an incentive to do the module. We therefore designed a certificate that nursing staff could put into their training portfolios. Feedback on those who completed the module was extremely positive.

PDSA cycle 4: Night nurses reported situations where fluid balance charts hadn't been reviewed during the day by ward doctors, leaving nurses unsure of whether to continue charts when they ran out at midnight. We fed this back at the weekly departmental meeting, to encourage ward doctors to ensure daily chart review. Instead of midnight-to-midnight monitoring, we changed to noon-tonoon monitoring, so that ward doctors would be present at the time of chart changeover.

PDSA cycle 5: Drawing on feedback from nursing staff, we made two further changes to the chart: addition of an "IV bolus" column, and removal of hourly cumulative totals in order to reduce workload. We facilitated access to calculators around the ward by attaching calculators to each of the mobile HR/BP monitors, and displayed posters showing the volumes of fluid in hospital drinks containers (figure 3).

PDSA cycle 6: We continued to use our final version of the fluid balance chart, which had become widely accepted by the ward. Post-intervention measurement was carried out at week 20 .

\section{Results}

We performed regular audit at the end of each PDSA cycle (approximately every three weeks), measuring the following parameters:

- Percentage of patients on fluid balance monitoring (FBM)

- Percentage of patients on FBM unnecessarily (ie no clinical indication)

- Average chart completion rate

- Average chart accuracy (see figure 4: results).

Post-intervention measurement (week 20) showed: 
- $38 \%$ of patients on FBM (a $43 \%$ reduction)

- 3\% of patients on FBM unnecessarily (a 93\% reduction)

- $70 \%$ average chart completion (a $40 \%$ increase)

- $61 \%$ average chart accuracy (a $48 \%$ increase).

Feedback from a survey regarding the e-Learning module ( $\mathrm{n}=81$ nurses and HCAs) showed that:

- $100 \%$ rated the usefulness of the module as 5 out of 5

- $96 \%$ gave it a score of 5 out of 5 for increasing understanding of the importance of fluid balance and the consequences of poor FBM.

See supplementary file: ds6180.pptx - "Figures"

\section{Lessons and limitations}

The main lesson we will take away from this project is how difficult it is to bring about a behavioural change in staff on a busy hospital ward, and the importance of education in making such a change succeed.

We initially made the mistake of changing the paperwork (making a new fluid chart), and expecting staff to automatically adapt to this. We did not provide enough information on the project in its early stages. Some staff resented the change, others were confused by it - this is reflected in the initial drop that we measured in chart completion and accuracy.

Our project truly began to grow once we addressed the issue of awareness and education: posters, presentations at nursing handover, e-Learning, and simply being present on the ward and reinforcing our message by speaking to staff. Thanks to these measures, we have succeeded in raising the profile of fluid balance monitoring on our ward. This is what has led to the change in people's behaviours - not the new paperwork. We have learned that behavioural change requires education first, and new paperwork later.

Thus, the human factor has been a large part of the success of our quality improvement project. From this, however, stems the main limitation of our project: the small sample size. Our interventions were labour-intensive: raising awareness and education requires constant reinforcement of the message through multiple channels, and a large dependence on human relations. These changes were possible because we had a dedicated QI team working on a relatively small environment (a thirty-bedded ward). We have yet to show whether these changes would be feasible to bring about at the scale of an entire hospital.

Another limitation of our project is its narrow scope: we chose to focus solely on education of nursing staff. Lack of knowledge around fluid balance and prescribing is also an issue which affects junior doctors themselves, especially in their first few months of hospital work following graduation from medical school. Our project did not address education of junior doctors, which would be the logical next step to overall improvement in fluid status management.

\section{Conclusion}

Inaccurate fluid balance monitoring is a well-known problem on all hospital wards that can have significant impacts on patient safety. Fluid balance monitoring can be improved by the implementation of a system where daily medical review of fluid charts allows rationalisation of monitoring, reducing unnecessary workload and improving chart completion and accuracy in patients who need it. In order to make such a cultural change succeed on a ward, it is crucial to raise awareness and education around the importance of fluid balance.

\section{References}

1 Boylan A, Brown P. Student observations. Fluid balance charts. Nurs Times. 1985;81(20):35-8.

2 Daffurn K, Hillman KM, Bauman A, Lum M, Crispin C, Ince L. Fluid balance charts: do they measure up? Br J Nurs. $1994 ; 3(16): 816-20$.

3 Perren A, Markmann M, Merlani G, Marone C, Merlani P. Fluid balance in critically ill patients. Should we really rely on it? Minerva Anestesiol. 2011 Aug;77(8):802-11.

4 NICE guidelines [CG174, Dec 2013] - Intravenous fluid therapy in adults in hospital.

5 Leach RM, Brotherton A, Stroud M, Thompson R. Nutrition and fluid balance must be taken seriously. BMJ. 2013 Feb 8;346:f801.

\section{Declaration of interests}

Nothing to declare.

\section{Acknowledgements}

We would like to thank Sister Helen Potter for her support in implementing our project on Coleridge Ward at Musgrove Park Hospital, William Bladon for his help in creating the e-Learning module, Miss Morrison and Pippa Richards for their help in promoting use of the e-Learning module amongst nursing staff, and all the junior doctors who helped in coordinating the project (Jemma Winterbottom, Julia Myers, Samuel Nava, and Rushan Perera).

\section{Ethical approval}

Ethical approval was not sought, as this was an improvement study focusing on systems for patient monitoring, not a study of human subjects. 